\title{
ГРАНИЧНІ ВИПАДКИ КРИТЕРІЮ МІНІМУМУ ПРОТЯЖНОСТІ
}

Анотація. Представлені граничні випадки критерію мінімуму протяжності, які відбивають його зв'язок з відомими критеріями обробки даних. Установлені відповідності між критерісм мінімуму протяжності й критеріями найменших квадратів, найменших модулів, максимуму правдоподібності в задачі оцінювання параметра зсуву за умови фіксованого параметра масштабу для незалежних однаково розподілених випадкових величин із законами розподілу Гаусса, Лапласа, Коші, "гостровершинним" меридіанним законом розподілу, законами узагальнених розподілів Гаусса й Коші, критерієм узагальненої максимальної правдоподібності з вартісними функціями Мішалкіна й Демиденка, а також критеріїм максимуму гістограми. Крім цього, у рамках концепції функціонала протяжності представлено кілька варіантів граничного переходу від критерію мінімуму квазіпротяжності до критерію мінімуму строгої протяжності, де останній критерій для дискретного випадку дає постановку NP-складної задачі мінімізації квазінорми простору 10. Підкреслено, що більшість із зазначених критеріїв приводить до постановки задач оптимізації з неопуклою та неунімодальною цільовою функцією. Ключові слова: критерій, протяжність, оптимізація.

Вступ. Критерій мінімуму протяжності [1] доцільно використовувати для постановки задач обробки даних, отримуваних в умовах складного шумового оточення й поданих параметричними або непараметричними моделями [2, 3]. Насамперед, це обумовлене широкими можливостями "м'якого" визначення поняття протяжності абстрактної функції, яке вбране у форму функціонала квазіпротяжності та побудоване на основі супермножини вартісних функцій. В [4] наведені деякі граничні випадки цієї супермножини, які дозволяють установлювати відповідні граничні випадки критерію мінімуму протяжності. У даній роботі підсумовуються результати виконання різних граничних переходів, які для критерію мінімуму протяжності здійснюються шляхом спрямовування значень віль-

(C) Вовк C.M., 2020 
«Системні технології» 1 (126) 2020 «System technologies»

них параметрів супермножини до їхніх граничних або особливих значень.

Постановка задачі та мета досліджень. Постановка задачі полягає в одержанні граничних випадків критерію мінімуму протяжності та зіставленні їх з відомими критеріями обробки даних. Метою даної роботи є встановлення відповідностей між критерієм мінімуму протяжності й іншими критеріями обробки даних.

Аналіз останніх досліджень i публікацій. Згідно 3 [1], протяжність абстрактної функції $f(x)$ доцільно подавати у двох формах, а саме у формі поняття “строга протяжність”:

$$
E[f(x)]=\int_{-\infty}^{\infty} \chi[f(x)] d x
$$

де $\chi[f(x)]=\left\{\begin{array}{ll}1 ; & f(x) \neq 0 \\ 0 ; & f(x)=0\end{array}\right.$, та у формі поняття “квазіпротяжність”:

$$
\begin{gathered}
E^{(\alpha, \beta, q)}[f(x)]=\int_{-\infty}^{\infty} \psi_{S}^{(\alpha, \beta, q)}[f(x)] d x ; \\
\psi_{S}^{(\alpha, \beta, q)}(x)=k_{S}^{(\alpha, \beta, q)} \cdot\left[\left(1+|x / \alpha|^{q}\right)^{\beta / q}-1\right],
\end{gathered}
$$

де $\alpha, q, \beta$ - вільно налаштовувані параметри, що задають величину згладжування, ступінь згладжування й форму функції, відповідно, причому $\alpha>0,0<q<\infty,-\infty<\beta \leq 1$ й $\beta<q ; k_{s}^{(\alpha, \beta, q)}=1 /\left[\left(1+\left|x_{0} / \alpha\right|^{q}\right)^{\beta / q}-1\right] ; x_{0}-$ точка нормування (3) на одиницю [1]. Поняття “квазіпротяжність” узагальнює поняття “строга протяжність”, бо з (2) випливає (1) [1].

Критерій мінімуму протяжності полягає у вимозі мінімізувати протяжність функції, використовуваної для пошуку розв'язку [1]. Застосування цього критерію до відхилу між даними $g(x)$ та їх моделлю $s(x ; \theta)$, що залежить від невідомого параметра $\theta$, призводить у дійсному неперервному випадку до задачі [2]:

$$
\min _{\theta} E^{(\alpha, \beta, q)}[s(x ; \theta)]=\min _{\theta}\left\{k^{(\alpha, \beta, q)} \cdot \int_{-\infty}^{\infty}\left[\left(1+|[g(x)-s(x ; \theta)] / \alpha|^{q}\right)^{\beta / q}-1\right] d x\right\} .
$$

У припущенні, що $\theta \epsilon$ лінійним параметром $A$, тобто $s(x ; A)=A f(x)$ та майже всюди $f(x) \neq 0$, задача (4) для дискретного випадку приймає вигляд: 
«Системні технологіï» 1 (126) 2020 «System technologies»

$$
\min _{A}\left\{k^{(\alpha, \beta, q)} \cdot \sum_{n=1}^{N}\left[\left(1+\left|\left[g_{n}-A f_{n}\right] / \alpha\right|^{q}\right)^{\beta / q}-1\right]\right\},
$$

де $g_{n}=g\left(x_{n}\right)$ - елементи даних, $f_{n}=f\left(x_{n}\right)$ - елементи моделі, яка 3 точністю до невідомого параметра $A$ описує дані, $n=1, \ldots, N$.

Критерій найменших квадратів за цих умов дає задачу [5]:

$$
\min _{A} \sum_{i=1}^{N}\left[g_{n}-A f_{n}\right]^{2}
$$

яка має очевидний розв'язок: $\hat{A}=\sum_{n=1}^{N}\left(g_{n} f_{n}\right) / \sum_{n=1}^{N} f_{n}^{2}$, тоді як критерій найменших модулів призводить до задачі [6]:

$$
\min _{A} \sum_{n=1}^{N}\left|g_{n}-A f_{n}\right|
$$

розв'язком якої $\epsilon$ зважена за допомогою величини $\left|f_{n}\right| \neq 0$ медіана значень $\left(g_{n} / f_{n}\right) ; n=1, \ldots, N$, тобто $\hat{A}=\operatorname{med}\left\{\left.\left|f_{n}\right| \circ\left(g_{n} / f_{n}\right)\right|_{n=1} ^{N}\right\}$, де ० позначає операцію тиражування $\left|f_{n}\right|$ раз значень $\left(g_{n} / f_{n}\right)$.

Критерій максимальної правдоподібності [7]:

$$
\max _{A} p\left(e_{1}, \ldots, e_{N} \mid A\right)=\max _{A} \ln p\left(e_{1}, \ldots, e_{N} \mid A\right),
$$

де $p\left(e_{1}, \ldots, e_{N} \mid A\right)$ - спільна щільність імовірності значень $e_{n}=g_{n}-A f_{n}$, дає в рамках проблеми оцінювання параметра зсуву за умови фіксованого параметра масштабу наступні задачі. Якщо всі $e_{n} ; n=1, \ldots, N \in$ незалежними однаково розподіленими (н.о.р.) за законом Гаусса зі скінченною дисперсією випадковими величинами (в.в.), то з (8) одержуємо (6). Якщо в цьому випадку законом розподілу є закон Лапласа, то з (8) одержуємо (7), а якщо цим законом є закон Коші з апріорно відомим параметром масштабу $s>0$, то маємо задачу [8]:

$$
\min _{A} \sum_{n=1}^{N} \ln \left(1+\left|g_{n}-A f_{n}\right|^{2} / s^{2}\right) .
$$

Для "гостровершинного" меридіанного закону розподілу н.о.р.в.в. $з$ відомим параметром масштабу $s>0$ маємо задачу [6]:

$$
\min _{A} \sum_{n=1}^{N} \ln \left(1+\left|g_{n}-A f_{n}\right| / s\right) .
$$

Для закону узагальненого розподілу Гаусса н.о.р.в.в. зі скінченною дисперсією й відомим параметром форми $0<\tau<\infty$ маємо задачу [8]: 
«Системні технологіï» 1 (126) 2020 «System technologies»

$$
\min _{A} \sum_{n=1}^{N}\left|g_{n}-A f_{n}\right|^{\tau}
$$

а для закону узагальненого розподілу Коші н.о.р.в.в. 3 апріорно відомими параметрами масштабу $s>0$ й "важкості хвостів" $0<t \leqslant 2$ маємо задачу [8]:

$$
\min _{A} \sum_{n=1}^{N} \ln \left(1+\left|g_{n}-A f_{n}\right|^{t} / s^{t}\right) \text {. }
$$

Критерій узагальненої максимальної правдоподібності є [9]:

$$
\min _{\theta} \sum_{n=1}^{N} \rho\left[y_{n} ; \theta\right]
$$

де $\rho\left[y_{n} ; \theta\right]$ - задана вартісна функція значень $y_{n}$, що залежать від невідомого параметра $\theta$. Для зазначених вище умов з (13) маємо задачу:

$$
\min _{A} \sum_{n=1}^{N} \rho\left[g_{n}-A f_{n}\right]
$$

Задаючи вартісну функцію Мєшалкіна [10]:

$$
\rho(x)=k_{M}\left[1-\exp \left(-\lambda x^{2} / 2\right)\right] ; \lambda>0,
$$

де $k_{M}=1 /[1-\exp (-\lambda / 2)], 3(14)$ маємо задачу:

$$
\min _{A} \sum_{n=1}^{N}\left[1-\exp \left(-\lambda\left|g_{n}-A f_{n}\right|^{2} / 2\right)\right]=\max _{A} \sum_{n=1}^{N} \exp \left(-\lambda\left|g_{n}-A f_{n}\right|^{2} / 2\right) \text {. }
$$

Задаючи вартісну функцію Демиденка [11]:

$$
\rho(x)=k_{D}\left[x^{2} /\left(x^{2}+c\right)\right] ; \quad c>0,
$$

де $k_{D}=1+c, 3(14)$ маємо задачу:

$$
\min _{A} \sum_{n=1}^{N}\left[\left|g_{n}-A f_{n}\right|^{2} /\left(\left|g_{n}-A f_{n}\right|^{2}+c\right)\right]=\max _{A} \sum_{n=1}^{N}\left(\left|g_{n}-A f_{n}\right|^{2}+c\right)^{-1} \text {. }
$$

Основна частина. Розв'язок поставленої задачі досягається шляхом спрямовування значень вільних параметрів функціонала квазіпротяжності до їхніх граничних або особливих значень. Далі розглянемо задачу (5) та її зв'язок з (6), (7), (9), (10), (11), (12), (16), (18).

Виконуючи граничний перехід за $\beta \rightarrow \pm 0$, з (5) отримуємо задачу:

$$
\min _{A} \sum_{n=1}^{N} \ln \left(1+\left|\left[g_{n}-A f_{n}\right] / \alpha\right|^{q}\right) \text {. }
$$

Легко бачити, що (19) збігається з (12) у випадку, коли $\alpha=s$ й $q=t$. Крім цього, (19) збігається з (9) у випадку $\alpha=s$ й $q=2$, а також збігається з (10) у випадку $\alpha=s$ й $q=1$. Слід зазначити, що цільова функція задачі (19) не $\epsilon$ 
«Системні технологіï» 1 (126) 2020 «System technologies»

опуклою та не є унімодальною за винятком тих граничних випадків, коли $\alpha \rightarrow \infty$ Й $q \geq 1$.

3 (5) шляхом граничного переходу за $\alpha \rightarrow \infty$ отримуємо задачу:

$$
\min _{A} \sum_{i=1}^{N}\left|g_{n}-A f_{n}\right|^{q}
$$

де $0<q<\infty$. Легко бачити, що (20) збігається з (11) у випадку $q=\tau$. Крім цього, (20) збігається з (6) у випадку $q=2$, а також збігається з (7) у випадку $q=1$. Цільова функція задачі (20) $є$ опуклою для $1 \leq q<\infty$ (у цих випадках вона описує норму нормованого простору $\left.l_{q} ; 1 \leq q<\infty\right)$ та не $\epsilon$ опуклою для $0<q<1$ (У цих випадках вона описує квазінорму ненормованого простору $\left.l_{q} ; 0<q<1\right)$.

Покладаючи в (5) $q=2$ й $\beta=-2$, а також враховуючи, що $\left.k_{S}^{(\alpha, \beta, q)}\right|_{\beta=-2 ; q=2}=1 /\left[\left(1+\left|x_{0} / \alpha\right|^{2}\right)^{-1}-1\right]=-\left[1+1 /\left|x_{0} / \alpha\right|^{2}\right]<0,3$ (5) отримуємо задачу:

$$
\min _{A}\left\{-\sum_{n=1}^{N}\left[\left(1+\left|\left[g_{n}-A f_{n}\right] / \alpha\right|^{2}\right)^{-1}-1\right]\right\}=\max _{A}\left\{\sum_{n=1}^{N}\left(\alpha^{2}+\left|g_{n}-A f_{n}\right|^{2}\right)^{-1},\right.
$$

що збігається 3 (18) для $c=\alpha^{2}$. Крім цього, покладаючи в (5) $q=2$ та $\alpha^{2}=(-\beta / 2) \alpha_{M}^{2}$, де $\alpha_{M} \epsilon$ параметром, що отримується для $x_{0}=1$ в результаті розв'язку рівняння [4]: $\alpha^{2} \ln \left[1+1 / \alpha^{2}\right]=\alpha_{M}^{2}\left[1-\exp \left(-1 / \alpha_{M}^{2}\right)\right], \quad 3$ (5) шляхом граничного переходу за $\beta \rightarrow-\infty$ отримуємо задачу:

$$
\min _{A} \sum_{n=1}^{N}\left[1-\exp \left(-\left|g_{n}-A f_{n}\right|^{2} / \alpha_{M}^{2}\right)\right]=\max _{A} \sum_{n=1}^{N} \exp \left(-\left|g_{n}-A f_{n}\right|^{2} / \alpha_{M}^{2}\right),
$$

що збігається з (16) для $\lambda=2 / \alpha_{M}^{2}$. Таким чином, (21) і (22) встановлюють відповідність між критерієм мінімуму протяжності й критерієм узагальненої максимальної правдоподібності з вартісними функціями Демиденка й Мєшалкіна, відповідно.

Якщо в (5) покласти $\alpha^{q}=(-\beta / q) \alpha_{g M}^{q}$, де значення параметра $\alpha_{g M}$ отримується для $x_{0}=1$ в результаті розв'язку рівняння [4]: $\alpha^{q} \ln \left[1+1 / \alpha^{q}\right]=\alpha_{g M}^{q}\left[1-\exp \left(-1 / \alpha_{g M}^{q}\right)\right]$, то 3 (5) шляхом граничного переходу за $\beta \rightarrow-\infty$ отримуємо задачу:

$$
\max _{A} \sum_{n=1}^{N} \exp \left(-\left|g_{n}-A f_{n}\right|^{q} / \alpha_{g M}^{q}\right) \text {. }
$$


«Системні технології» 1 (126) 2020 «System technologies»

Спрямовуючи $q \rightarrow \infty$, з (23) отримуємо задачу:

$$
\max _{A} \sum_{n=1}^{N} \operatorname{rect}\left[\left|g_{n}-A f_{n}\right| /\left(2 \alpha_{g M}\right)\right]
$$

де $\quad \operatorname{rect}[x /(2 \alpha)]=1 \quad$ для $|x|<\alpha ; \quad \operatorname{rect}[x /(2 \alpha)]=e^{-1} \approx 0,368 \quad$ для $|x|=\alpha$; $\operatorname{rect}[x /(2 \alpha)]=0$ для $|x|>\alpha$. Покладаючи для спрощення $f_{n}=1 ; n=1, \ldots, N, 3$ (24) одержуємо задачу пошуку максимуму за невідомим параметром $A$ гістограми значень $g_{n} ; n=1, \ldots, N$ із шириною колодязів $2 \alpha_{g M}$.

Граничні переходи від критерію мінімуму квазіпротяжності до критерію мінімуму строгої протяжності отримуються під час перетворення функціонала квазіпротяжності $E^{(\alpha, \beta, q)}$ у функціонал строгої протяжності $E$. Для випадку використання звичайного параметра $\alpha$ можна записати такі співвідношення (для їхнього одержання важливу роль відіграє коефіцієнт $\left.k_{S}^{(\alpha, \beta, q)}=1 /\left[\left(1+\left|x_{0} / \alpha\right|^{q}\right)^{\beta / q}-1\right]\right)$ :

- для $-\infty<\beta<0 є$ вірним: $\lim _{\alpha \rightarrow 0} E^{(\alpha, \beta, q)}=E$, а також $\lim _{\beta \rightarrow 0}\left(\lim _{\alpha \rightarrow 0} E^{(\alpha, \beta, q)}\right)=E$;

- для $\alpha>0$ и $0<q<\infty$ виконується: $\lim _{\beta \rightarrow-\infty} E^{(\alpha, \beta, q)}=E$;

- для $0<q<\infty$ виконується: $\lim _{\alpha \rightarrow 0}\left(\lim _{\beta \rightarrow 0} E^{(\alpha, \beta, q)}\right)=E$;

- для $\alpha>0$ виконується: $\lim _{q \rightarrow 0}\left(\lim _{\beta \rightarrow 0} E^{(\alpha, \beta, q)}\right)=E$;

- для $-\infty<\beta<1$ виконується: $\lim _{q \rightarrow 0}\left(\lim _{\alpha \rightarrow \infty} E^{(\alpha, \beta, q)}\right)=E$.

Для випадку використання модифікованого параметра $\alpha_{g м}$ можна записати такі співвідношення:

- для $0<q<\infty$ виконується: $\lim _{\alpha_{g M} \rightarrow 0}\left(\lim _{\beta \rightarrow-\infty} E^{\left(\alpha_{g M}, \beta, q\right)}\right)=E$;

- для $\alpha_{g M}>0$ виконується: $\lim _{q \rightarrow 0}\left(\lim _{\beta \rightarrow-\infty} E^{\left(\alpha_{g M}, \beta, q\right)}\right)=E$.

Таким чином, існують різні варіанти зазначеного граничного переходу. Слід також зауважити, що для дискретного випадку мінімізація строгої протяжності скінченної послідовності даних означає мінімізацію кількості її ненульових елементів або, зі зворотної сторони, максимізацію кількості її нульових елементів.

3 поданого в цій роботі розгляду випливає, що представлені результати не залежать від виду й параметрів математичної моделі даних. Це обумовлене тим, що зазначені граничні випадки критерію мінімуму 42 
«Системні технологіï» 1 (126) 2020 «System technologies»

протяжності отримуються шляхом граничних переходів функціонала квазіпротяжності за його вільними параметрами.

Висновки. В поданій роботі установлені відповідності між критерієм мінімуму протяжності та іншими критеріями обробки даних. Отримані граничні випадки критерію мінімуму протяжності дозволяють зробити висновок про те, що критерій мінімуму протяжності може використовуватися як універсальний інструмент з постановки задач моделювання й обробки даних для різних умов їх спостереження.

\section{ЛІТЕРАТУРА / ЛИТЕРАТУРА}

1. Вовк С. М. Критерій мінімуму протяжності / С. М. Вовк // Системні технології. Регіональний міжвузівський збірник наукових праць. Випуск 1 (120). - Дніпро, 2019. - С. 19 - 25.

2. Вовк С. М. Постановка задач обработки данных на основе критерия минимума протяженности / С. М. Вовк // Радіоелектроніка, інформатика, управління. - 2019. - N.1 -C. 157-166.

3. Вовк С. М. Метод обробки даних в умовах складного шумового оточення / С. М. Вовк, В. В. Гнатушенко // Науковий вісник Таврійського державного агротехнологічного університету. -2018. - Вип. 8. - Т.2. - С. 1-9. 4. Vovk S.M. General approach to building the methods of filtering based on the minimum duration principle / S. M. Vovk // Radioelectronics and Communications Systems. - 2016. - V. 59. - N. 7 - P. 281-292.

5. Wolberg J. Data Analysis Using the Method of Least Squares: Extracting the Most Information from Experiments / J. Wolberg. - Berlin: Springer-Verlag, 2005. $-250 \mathrm{p}$.

6. Aysal T. C. Meridian filtering for robust signal processing / T. C. Aysal, K. E. Barner // IEEE Tr. Signal Processing. - 2007. - V. 55. - N. 8. - P. 3949-3962.

7. Millar R. B. Maximum Likelihood Estimation and Inference: With Examples in R, SAS and ADMB / R. B. Millar. - New York: Wiley, 2011.-376 p.

8. Carrillo R. E. Generalized Cauchy distribution based robust estimation / R.

E. Carrillo, T. C. Aysal, K. E. Barner // Proc. of Int. Conf. Acoustic, Speech and Signal Processing, ICASSP 2008, Las Vegas, 2008. - P. 3389-3392.

9. Huber P. Robust statistics. 2nd ed. / P. Huber, E. M. Ronchetti. - Hoboken: Wiley, 2009. - 370 p.

10. Shevlyakov G. L. Robustness in data analysis: criteria and methods / G. L. Shevlyakov, N. O. Vilchevski. - Utrecht: VSP. - 2002. - 310 p. 
«Системні технології» 1 (126) 2020 «System technologies»

11. Демиденко Е. 3. Оптимизация и регрессия / Е. 3. Демиденко. - М.: Наука, 1989. - 296 с.

\section{REFERENCES}

1. Vovk S. M. Kryterii minimumu protiazhnosti / S. M. Vovk // Systemni tekhnolohii. Rehionalnyi mizhvuzivskyi zbirnyk naukovykh prats. Vypusk 1 (120). - Dnipro, 2019. - C. 19 - 25.

2. Vovk S. M. Postanovka zadach obrabotki dannyih na osnove kriteriya minimuma protyazhennosti / S. M. Vovk // Radioelektronika, informatyka, upravlinnia. - 2019. - N.1 - S. 157-166.

3. Vovk S. M. Metod obrobky danykh v umovakh skladnoho shumovoho otochennia / S. M. Vovk, V. V. Hnatushenko // Naukovyi visnyk Tavriiskoho derzhavnoho ahrotekhnolohichnoho universytetu. -2018. -Vyp. 8. - T.2. - S. 1-9. 4. Vovk S.M. General approach to building the methods of filtering based on the minimum duration principle / S. M. Vovk // Radioelectronics and Communications Systems. - 2016. - V. 59. - N. 7 - P. 281-292.

5. Wolberg J. Data Analysis Using the Method of Least Squares: Extracting the Most Information from Experiments / J. Wolberg. - Berlin: Springer-Verlag, 2005. $-250 \mathrm{p}$.

6. Aysal T. C. Meridian filtering for robust signal processing / T. C. Aysal, K. E. Barner // IEEE Tr. Signal Processing. - 2007. - V. 55. - N. 8. - P. 3949-3962.

7. Millar R. B. Maximum Likelihood Estimation and Inference: With Examples in R, SAS and ADMB / R. B. Millar. - New York: Wiley, 2011.- 376 p.

8. Carrillo R. E. Generalized Cauchy distribution based robust estimation / R. E. Carrillo, T. C. Aysal, K. E. Barner // Proc. of Int. Conf. Acoustic, Speech and Signal Processing, ICASSP 2008, Las Vegas, 2008. - P. 3389-3392.

9. Huber P. Robust statistics. 2nd ed. / P. Huber, E. M. Ronchetti. - Hoboken: Wiley, 2009. - $370 \mathrm{p}$.

10. Shevlyakov G. L. Robustness in data analysis: criteria and methods / G. L. Shevlyakov, N. O. Vilchevski. - Utrecht: VSP. - 2002. - 310 p.

11. Demidenko E. Z. Optimizatsiya i regressiya / E. Z. Demidenko. - M.: Nauka. - 1989. - 296 s.

Received 09.01.2020. Accepted 15.01.2020.

\section{Граничні випадки критерію мінімуму протяжності}

Розглянуті граничні випадки критерію мінімуму протяжності та установлені відповідності між критерієм мінімуму протяжності й критеріями найменших квадратів, найменших модулів, максимуму правдоподібності для незалежних однаково розподілених випадкових величин із законами розподілу Гаусса, Лапласа, Коші, меридіанним законом 44 


\section{«Системні технологіï» 1 (126) 2020 «System technologies»}

розподілу, законами узагальнених розподілів Гаусса й Коші, критерієм узагальненої максимальної правдоподібності з вартісними функціями Мєшалкіна й Демиденка, а також критерієм максимуму гістограми. Представлені варіанти граничного переходу від критерію мінімуму квазіпротяжності до критерію мінімуму строгої протяжності. Підкреслено, що більшість із зазначених критеріїв призводить до задач оптимізації з неопуклою та неунімодальною цільовою функцією.

\section{Limiting cases of criterion of minimum-extent}

The minimum-extent criterion is appropriate to use for the problem formulation of processing of data obtained with a complicated noise environment and represented by parametric or nonparametric models. In this paper, the various limiting cases of minimum-extent criterion, which are carried out by tending the values of its three free parameters to their limiting or special values, are summarized. The goal of this paper is to obtain the relations between the minimum-extent criterion and other data processing criteria.

By calculating the corresponding limits, there are obtained the relations between the minimum-extent criterion and the following criteria. Among these criteria there are the least squares criterion, the least absolute deviations criterion, the maximum likelihood criterion in the problem of shift parameter estimating with a fixed scale parameter for independent identicallydistributed random variables with the generalized Gaussian distribution and the generalized Cauchy distribution, the generalized maximum likelihood criterion with the Meshalkin's and Demidenko's cost functions, as well as the criterion of the maximum of the histogram. In addition, the several variants of the limiting passage from the criterion of minimum of the quasiextent to the criterion of minimum of the strict extent are presented, where the latter gives the statement of the NP-hard problem of minimizing the quasi-norm of 10-space for the discrete case. It is emphasized that most of these criteria lead to the formulation of the optimization problem with a non-convex and non-unimodal objective function. It is also indicated that the presented results do not depend on the type and parameters of the used mathematical data model. The obtained limiting cases allow to conclude that the minimum-extent criterion is an universal tool to formulate the problems of data modeling and data processing for different conditions of data observation.

Вовк Сергей Михайлович - к.ф.-м.н., доцент, Днепровский национальный университет имени О.Гончара, доцент кафедры компьютерных наук и информационных технологий.

Вовк Сергій Михайлович - к.ф.-м.н., доцент, Дніпровський національний університет імені О.Гончара, доцент кафедри комп'ютерних наук та інформаційних технологій.

Vovk Serhii - Ph.D, Associate Professor, Oles Honchar Dnipro National University, Associate Professor of Department of Computer Science and Information Technology. 